
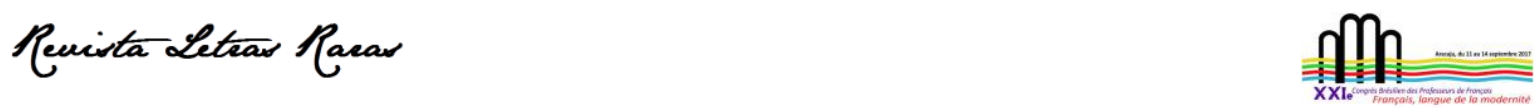

Actes du XXlème Congrès Brésilien des Professeurs De Français dans Édition spéciale de la Revue Letras Raras, 2021

ISSN : 2317-2347 - v. 10, Dossier Spécial (2021)

Todo o conteúdo da RLR está licenciado sob Creative Commons Atribuição 4.0 Internacional

\title{
Systèmes juridiques français et brésilien: enjeux de la conception des cours français du droit
}

\section{Pedro Armando de Almeida Magalhães *}

Professeur de Français à l'Institut de Lettres de l'Université de l'Etat de Rio de Janeiro ; Premier sécretaire à la FBPF ; Directeur à l'APFERJ ; chercheur dans le domaine de l'enseignement/apprentissage des langues/cultures et de la littérature comparée (rapports entre le discours de la fiction et le discours de l'Histoire).

\section{iD https://orcid.org/0000-0002-1137-7052}

Reçu en : 30 juin 2021. Approuvé en: 23 juillet 2021.

\section{Comment citer cet article:}

MAGALHÃES, Pedro Armando de Almeida. Systèmes juridiques français et brésilien: enjeux de la conception des cours français du droit. Revista Letras Raras, p. 206-216, n. Spécial, v. 10, nov. 2021.

\section{RÉSUMÉ}

La vogue des cours de français de spécialité et des cours de français sur objectif(s) spécifique(s) est certes étroitement liée à l'utilisation des TICE. Mais il faut analyser l'impact sur certaines modalités de formation, qui requièrent des connaissances approfondies dans certains domaines professionnels. Voilà pourquoi on choisira le cours FRANÇAIS DU DROIT pour étudier les enjeux de la conception de cours de français de spécialité. La bonne sélection des modules, l'étude attentive du lexique juridique et le choix raisonné des documents doivent prévaloir pour qu'on ne néglige pas l'aspect interculturel. En partant des travaux de didacticiens tels que Florence Mourlhon-Dallies, Jean-Marc Mangiante, Chantal Parpette et Christian Puren, nous proposons une réflexion sur la conception des cours français du droit en essayant de montrer un aperçu des différences les plus importantes entre le système juridique français et le système juridique brésilien. On signalera quelques particularités concernant les branches du droit, les juridictions judiciaires et les juridictions administratives, les professionnels du droit et les spécificités lexicales.

MOTS-CLÉS : Français du droit; français de spécialité; FOS; conception des cours

\section{En guise d'introduction - la question des méthodologies et des types de cours FLE}

De nos jours on vit une période où l'utilisation de différents médias, de différents outils informatiques, nous conduit à une inévitable remise en question des pratiques pédagogiques et des choix méthodologiques. Dans le domaine de l'enseignement/apprentissage du français langue

magalhaes.pedro67@outlook.com 

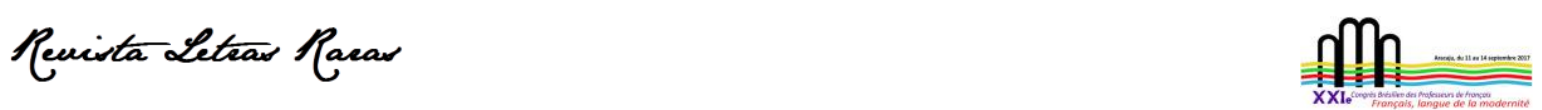

Actes du XXlème Congrès Brésilien des Professeurs De Français dans Édition spéciale de la Revue Letras Raras, 2021 ISSN : 2317-2347 - v. 10, Dossier Spécial (2021)

Todo o conteúdo da RLR está licenciado sob Creative Commons Atribuição 4.0 Internacional

étrangère on arrive au temps de l'éclectisme didactique, bien que la perspective actionnelle soit encore l'approche méthodologique la plus préconisée. L'éclectisme est un concept important pour la relation entre l'enseignant et l'apprenant, car il permet le brassage de plusieurs méthodologies, à des degrés différents. Tout dépend des besoins, ainsi que des objectifs visés. En effet, comprendre quels sont les besoins des publics-cibles dans toute leur hétérogénéité et leurs particularités aide l'enseignant à mieux concevoir les unités qui composeront la formation qu'il veut proposer.

C'est pourquoi, associé au concept d'éclectisme, on doit ajouter le concept de complexité, développé par le philosophe Edgar Morin (1994). Grâce aux innovations et à l'accès à l'information, on est capable de répondre aux défis du monde contemporain avec beaucoup plus d'acuité. Si les apprenants sont divers, les enseignants, pour leur part, peuvent cerner les spécificités humaines et, grâce aux ressources abondantes sur internet, concevoir des cours mieux adaptés.

Cette volonté de faire correspondre les besoins du public-cible à la définition des objectifs des cours a conduit, en quelque sorte, au développement des catégories de cours de français langue étrangère. Toutefois, on doit reconnaître que vouloir restreindre les objectifs est inévitable dans une certaine mesure dans les cours de FLE généralistes. En outre, on ne peut pas oublier que certains cours de FLE étaient instrumentaux, qualificatif désuet mais encore employé aujourd'hui pour désigner des cours qui ont pour but l'acquisition d'une compétence de compréhension écrite, dans la plupart des cas.

Alors, aujourd'hui on assiste à une profusion de cours de français de spécialité, ou de cours FOS (français sur objectifs spécifiques) ou de cours FOU (français sur objectifs universitaires) dans les écoles de langue et dans les universités (en raison des programmes d'échange et de la mobilité). La possibilité d'accès facile à des contenus spécifiques sur internet, le développement de la communication globale et des divers outils, logiciels, applications, ont permis cette progression et ont conduit inévitablement à une réflexion sur la conception des cours.

Il faut tout de même souligner que la distinction entre des cours de français de spécialité, des cours FOS et des cours FOU n'est pas toujours facile. On constate que ces trois catégories de cours dans le domaine de l'enseignement/apprentissage du FLE tendent parfois à se confondre et que les cours FOS, en tant qu'objet d'étude et de recherche, jouit d'une certaine préférence de la part des enseignants-chercheurs. 

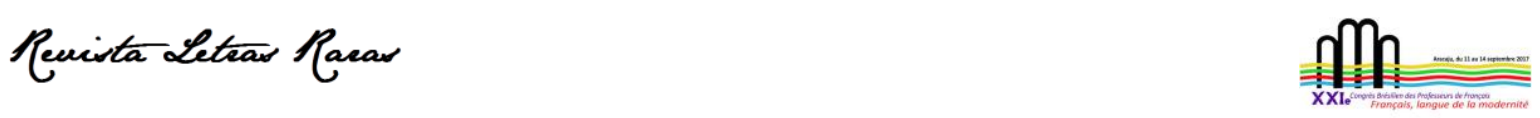

Actes du XXlème Congrès Brésilien des Professeurs De Français dans Édition spéciale de la Revue Letras Raras, 2021 ISSN : 2317-2347 - v. 10, Dossier Spécial (2021)

Todo o conteúdo da RLR está licenciado sob Creative Commons Atribuição 4.0 Internacional

C'est vrai que les principes observés dans la conception des cours FOS sont aussi utiles dans la conception des cours de français de spécialité ou des cours FOU. Toutefois, on remarque que la catégorie cours de français de spécialité est en quelque sorte engloutie par la popularité des cours FOS, alors que les cours FOU semblent être une catégorie de cours FOS.

Dans le présent travail on respectera la distinction de ces trois catégories de cours. Puisque on s'intéresse au cours français du droit, qui s'adresse au public de juristes, d'étudiants en droit et d'autres intéressés, on se propose de réfléchir à la conception des formations, même si l'on reconnaît l'existence de manuels spécialisés, dont l'utilité est prouvée. II faut tout de même souligner que ces réflexions sur la conception des cours français du droit sont aussi le fruit d'une expérience, une pratique répétée, mais toujours différente et enrichissante. Non seulement cette expérience je l'ai acquise dans les salles de l'Alliance Française à Rio de Janeiro, dans des cours au mois de juillet ou pendant un semestre, mais aussi dans une Université publique, dans le cadre d'un cours de français instrumental s'adressant à des étudiants en droit.

Bien sûr, on ne pourra pas ici aborder toute la complexité de la question de la conception de cours français du droit. L'objectif est de signaler certaines démarches possibles, à partir d'une réflexion didactique et didactologique. Comprendre les enjeux de la conception contribue à affronter les défis fréquents.

Le classement correct peut faciliter la conception, en nous signalant l'ordre et les spécificités des étapes à suivre. D'abord on cherchera à définir le cours français du droit. Ensuite on abordera la conception et quelques éléments à prendre en compte. Et à la fin on entrera dans le domaine de l'interculturel en énumérant quelques différences entre le système juridique français et le système juridique brésilien.

\section{Le cours français du droit: comment pourrait-on classer ce type de formation?}

Classer un cours n'est pas toujours évident. Et il est faux de croire que tous les cours français du droit appartiennent forcément à la catégorie des cours FOS au sens strict. II y a plusieurs éléments qui contribuent à une bonne définition du type de cours. Pouvoir classer correctement aide à mieux concevoir, à mieux structurer les unités pédagogiques. 

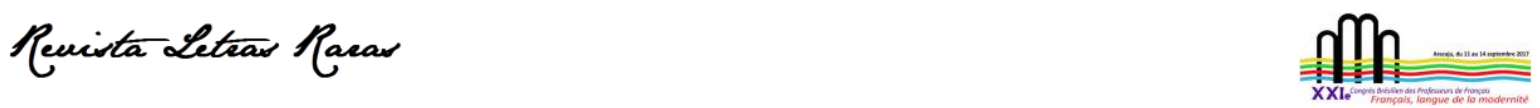

Actes du XXlème Congrès Brésilien des Professeurs De Français dans Édition spéciale de la Revue Letras Raras, 2021 ISSN : 2317-2347 - v. 10, Dossier Spécial (2021)

Todo o conteúdo da RLR está licenciado sob Creative Commons Atribuição 4.0 Internacional

\subsection{Cours français du droit: cours de français de spécialité ou de français sur objectifs spécifiques?}

Le cours français de spécialité est un cours offert par une institution ou un formateur sans qu'il y ait forcément une demande préalable spécifique. L'offre est l'élément principal. Le concepteur essaie de proposer une formation dans un domaine précis pour attirer un public désireux d'apprentissage du français juridique, médical, etc. La conception des modules et son séquençage correspond à un point de vue généraliste de la spécialité. Le cours français du droit offert par l'Alliance Française au début d'un semestre ou pendant le mois de juillet (mois de vacances) est un exemple de cours de français de spécialité.

En revanche, si l'institution reçoit une demande spécifique de la part d'un groupe de professionnels voulant développer certaines compétences dans leur domaine en français, il s'agit d'un cours de français sur objectifs spécifiques. Si, par exemple, un groupe d'avocats, spécialistes en droit pénal, désire un cours de perfectionnement pour mieux connaître le fonctionnement des juridictions judiciaires pénales, le concepteur choisira l'organisation et le contenu des modules les mieux adaptés aux besoins de ce public-cible, afin d'atteindre des objectifs très précis.

Donc, le cours français du droit peut être un cours de français de spécialité ou un cours de français sur objectifs spécifiques. Tout dépend de la conception de la formation. Il s'agit d'un cours offert à un public vaste de professionnels du droit (spécialistes de plusieurs branches)? Ou d'un cours répondant à une demande précise, conçu selon les besoins explicites d'un groupe spécifique de professionnels du droit?

\subsection{Cours FOU?}

De même, il se peut que le cours de français du droit soit offert dans une université. Dans ce cas, il peut s'adresser à des étudiants en droit désireux de perfectionner le français tout en développant des compétences langagières et académiques appartenant au domaine spécifique du droit. Alors il s'agirait plutôt d'un cours FOU pour les étudiants de la Faculté de Droit. Outre les particularités du domaine juridique que l'on doit observer, il faut penser à la culture propre à 

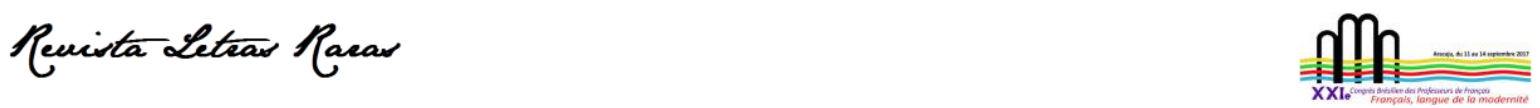

Actes du XXlème Congrès Brésilien des Professeurs De Français dans Édition spéciale de la Revue Letras Raras, 2021 ISSN : 2317-2347 - v. 10, Dossier Spécial (2021)

Todo o conteúdo da RLR está licenciado sob Creative Commons Atribuição 4.0 Internacional

l'université française, par exemple : structure des cours, débouchés, situations d'apprentissage spécifiques, techniques rédactionnelles, etc.

Savoir identifier le type de formation que l'on veut offrir est donc essentiel, pour mieux se préparer à la conception des cours.

\section{La conception des cours}

Aujourd'hui le rôle de l'enseignant est remis en question. L'enseignant ne joue plus le rôle de transmetteur de connaissances comme autrefois. Certes, c'est quelqu'un qui possède des savoirs précis, approfondis, mais l'accès libre à l'information grâce à l'internet modifie sa façon d'agir face aux apprenants. L'enseignant peut choisir des documents très actuels et doit perfectionner sa façon de concevoir des cours.

\subsection{L'utilisation des technologies de l'information et de la communication}

On ne peut pas se passer des TICE lorsque l'on se propose de concevoir des formations de français du droit. D'abord parce qu'elles sont une excellente source de données actuelles. Puis parce que le droit peut subir des changements importants (nouvelles lois, nouvelle jurisprudence, nouvelles institutions). Toutefois, le choix des documents sur internet exige une certaine habileté et prudence. Bien sûr, on doit vérifier la source et la provenance des documents avant de les choisir. Par exemple, un document sur Wikipédia n'a pas la même valeur qu'un article juridique diffusé dans le site du Ministère de la Justice français. Et même si on dispose de bons manuels de français du droit, ils n'arrivent pas à remplir les lacunes que l'analyse des besoins dévoile. D'une part, les manuels n'arrivent pas à suivre les changements survenus dans les systèmes juridiques. D'autre part, le public des apprenants est souvent composé de spécialistes intéressés, désireux de comprendre les détails et les actualités du droit. Alors, puisque la part dévolue aux ressources internet reste importante, c'est à l'enseignant de faire le tri consciencieux. 

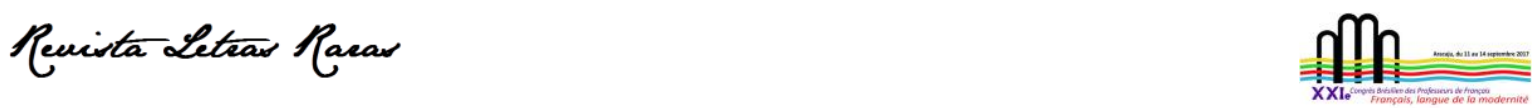

Actes du XXlème Congrès Brésilien des Professeurs De Français dans Édition spéciale de la Revue Letras Raras, 2021 ISSN : 2317-2347 - v. 10, Dossier Spécial (2021)

Todo o conteúdo da RLR está licenciado sob Creative Commons Atribuição 4.0 Internacional

\subsection{Public de professionnels du droit et d'étudiants en droit: des besoins précis; urgence de la demande. Complexité dans la définition des objectifs.}

Face à un public composé de juristes et d'étudiants pour la plupart, les besoins sont souvent définis selon les branches préférées. L'enseignant n'est pas censé maîtriser autant de savoirs; il est plutôt la voie d'accès ou l'intermédiaire de contenus précis correspondant à des domaines complexes du droit. Voilà pourquoi il jouera plutôt le rôle de tuteur, encourageant ainsi l'exploitation de ressources variées et guidant les apprenants.

Selon qu'il s'agisse d'un cours de français de spécialité ou de FOS la démarche de l'enseignant peut varier : si le public est très diversifié, le contenu des cours sera diversifié, général ; si le public s'intéresse à une branche spécifique du droit, on augmentera la spécialisation du contenu, ce qui requiert un effort d'instrumentalisation et une certaine expertise didactique de la part de l'enseignant.

Et on ne peut pas nier que si l'enseignant possède des connaissances juridiques, une certaine culture du droit, cela aidera certainement à mieux façonner le séquençage des unités pédagogiques et à préciser les démarches à accomplir. Si, par contre, ce n'est pas le cas, il est souhaitable que l'enseignant puisse consulter des professionnels du droit avant et pendant la formation.

\subsection{L'importance des ressources bibliographiques, des sites et des dictionnaires}

Si l'on reste dans le domaine du droit français, on pourrait dire que deux manuels sont assez utiles lors de la préparation des cours: un ouvrage paru aux éditions CLÉ, intitulé Le français du droit (1998), de J.-L. Perfornis et un autre paru aux éditions Hachette, intitulé Le français juridique (2003), de Michel Soignet. Ces manuels peuvent servir de référence, mais ne suffisent pas dans la plupart des cas. D'abord, parce que le droit ne cesse de changer, si bien que l'enseignant doit toujours confirmer l'actualité des données. Ensuite parce que les ressources à disposition sur internet permettent une meilleure précision dans la correspondance entre les besoins et les objectifs visés. En ce qui concerne la conception il faut souligner que deux sites sont indispensables: www.justice.gouv.fr (site du Ministère de la Justice, qui explique l'ordre 

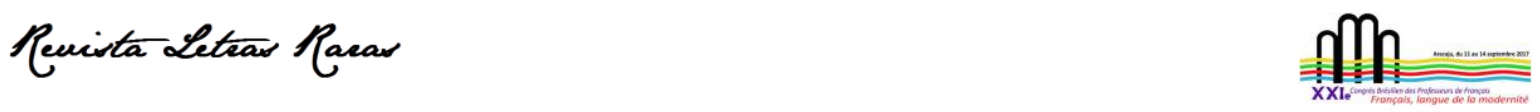

Actes du XXlème Congrès Brésilien des Professeurs De Français dans Édition spéciale de la Revue Letras Raras, 2021 ISSN : 2317-2347 - v. 10, Dossier Spécial (2021)

Todo o conteúdo da RLR está licenciado sob Creative Commons Atribuição 4.0 Internacional

judiciaire à l'aide d'organigrammes, textes et vidéos) et www.legifrance.gouv.fr qui diffuse les lois françaises et les traités européens. Quant aux dictionnaires, outre les documents lexicaux en ligne, des ouvrages en papier, tels que le Vocabulaire Juridique (2011), de Gérard Cornu, aux éditions PUF, peuvent être extrêmement utiles.

\subsection{L'interculturel: différences entre des systèmes différents. Grand défi à relever}

Le système juridique français est différent du système juridique brésilien, ce qui constitue une évidence, puisque la culture et l'histoire de chaque pays sont distinctes. Bien que le Code Civil français, appelé le Code Napoléon, ait inspiré le droit civil brésilien et que l'époque des Lumières (XVIIlème siècle) soit une référence pour le droit constitutionnel mondial, le droit change selon les pays ; les institutions ne sont pas les mêmes ; les coutumes et la jurisprudence sont hétérogènes.

Cependant, quand on compare le système français au système brésilien, en ce qui concerne les branches du droit, on remarque quand même assez de ressemblances, ce qui aide à la compréhension globale de l'organisation judiciaire française. Toutefois, quand on approfondit l'analyse, on remarque des distinctions structurelles à l'intérieur des juridictions judiciaires et dans la façon dont on distingue ces mêmes juridictions et les juridictions administratives. Quant aux professionnels, la correspondance entre la France et le Brésil est assez inégale. Par exemple: les termes avocat et juriste ne sont pas employés de la même manière. Et globalement, en ce qui concerne la terminologie, la forme discursive en France et au Brésil exige beaucoup de lecture et d'attention.

4 Quelques différences entre le système juridique français et le système juridique brésilien

Quand on se propose d'analyser les différences entre les deux systèmes on ne saurait être exhaustif. Voilà pourquoi il est préférable, dans cette étude préliminaire axée sur la conception des cours, de signaler quelques thèmes pertinents, qui méritent une étude approfondie. Voici quatre sujets qui suscitent un vif intérêt : 1) les branches du droit ; 2) les juridictions judiciaires et les juridictions administratives ; 3) Les professionnels du droit ; et 4) Les spécificités lexicales 

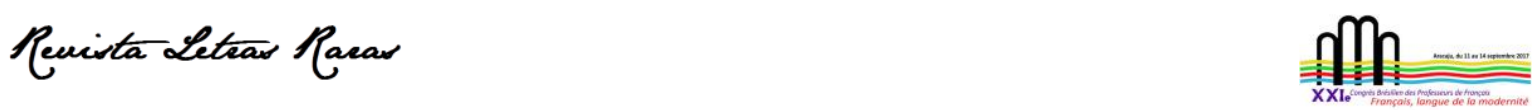

Actes du XXlème Congrès Brésilien des Professeurs De Français dans Édition spéciale de la Revue Letras Raras, 2021 ISSN : 2317-2347 - v. 10, Dossier Spécial (2021)

Todo o conteúdo da RLR está licenciado sob Creative Commons Atribuição 4.0 Internacional

\subsection{Les branches du droit}

II est très important de souligner, dès le début, que la ressemblance globale entre les domaines d'étude a pour effet de mettre en évidence les désaccords. Par exemple, si l'on se consacre à l'étude du droit constitutionnel, on dévoilera les différences entre les institutions et les régimes politiques français et brésilien. D'une part, la dimension territoriale des deux pays n'est pas la même, ce qui suggère que la gestion des territoires ne peut pas fonctionner de la même manière. D'autre part, le régime républicain français n'est pas conçu de la même façon que le régime républicain brésilien.

\subsection{Les juridictions judiciaires et les juridictions administratives}

Les juridictions judiciaires françaises n'ont pas la même configuration que celles du Brésil. Par exemple: dans les Tribunaux de Grande Instance en France, c'est un groupe de trois juges qui rendent le jugement, alors qu'au Brésil, à la première instance civile, il n'y a pas de forme collégiale à proprement parler. De même aux juridictions pénales, réparties en France selon le degré de l'infraction. Or, la distinction française entre délit et crime n'existe pas au Brésil.

Contrairement au Brésil, où les juridictions administratives sont assez hétérogènes et assez cachées dans leur fonctionnement, en France elles sont homogènes, bien structurées et composées de professionnels ayant suivi leurs études à l'ENA (Ecole Nationale d'Administration).

\subsection{Les professionnels du droit}

En ce qui concerne les noms de fonctions et leurs attributions il existe des différences qui sont assez importantes, sinon fondamentales. On en a choisi deux, à titre d'exemple :

\subsubsection{Différence entre juriste et avocat}

En France ceux qui ont suivi des cours de droit sont considérés comme des juristes. Au Brésil, par contre, seuls les savants ou penseurs du droit les plus éminents sont qualifiés de 

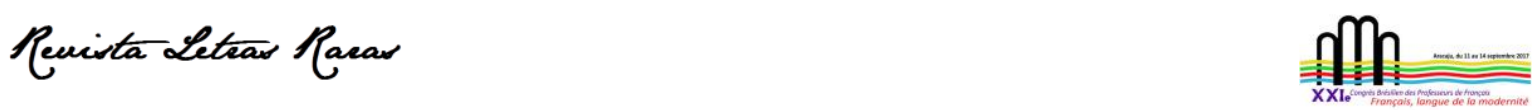

Actes du XXlème Congrès Brésilien des Professeurs De Français dans Édition spéciale de la Revue Letras Raras, 2021 ISSN : 2317-2347 - v. 10, Dossier Spécial (2021)

Todo o conteúdo da RLR está licenciado sob Creative Commons Atribuição 4.0 Internacional

juristas. En plus, en France, pour devenir avocat, il faut d'abord passer un concours après la Faculté de Droit à l'Ecole d'Avocats (EDA). Seuls ceux qui suivent le cours d'un an et demi dans cette école pourront devenir avocats. Au Brésil, au contraire, il suffit que les diplômés en Droit passent l'examen du Barreau des Avocats.

\subsubsection{Différence entre procureur et juge}

En France les procureurs intègrent la même carrière, celle de la magistrature, et ils ont le choix entre suivre celle de juge ou celle de procureur. En revanche, au Brésil les distinctions sont nombreuses, entre les membres du Ministère Public et entre le Ministère Public et la Magistrature. Les membres du Ministère Public au Brésil ne sont pas magistrats et sont répartis dans plusieurs filières professionnelles (Promotor de Justiça, Procurador do Município, Procurador do Estado, Procurador da República, etc.)

\subsection{Les spécificités lexicales}

Les spécificités lexicales sont nombreuses à l'oral et à l'écrit. Le langage juridique présente des particularités en France et au Brésil. Par exemple, en France, si on analyse le document d'un jugement rendu par un tribunal de grande instance, on remarquera les sections «FAITS ET PROCÉDURE », «SUR QUOI LE TRIBUNAL » et «PAR CES MOTIFS ». D'ailleurs, dans la deuxième section des jugements l'articulateur « attendu que [...]» est fréquent. En effet, la complexité du langage juridique est un aspect d'ordre technique qu'il faut absolument prendre en compte. Des termes qui peuvent sembler désuets ou rares en français standard ou en portugais courant sont souvent employés dans le milieu juridique.

\section{Conclusion}

Quelle que soit la modalité de la formation, français de spécialité, français sur objectifs spécifiques (FOS) ou français sur objectifs universitaires, l'enseignant-concepteur doit tenir compte des besoins du public-cible, afin de mieux établir les objectifs et ainsi organiser mieux le 

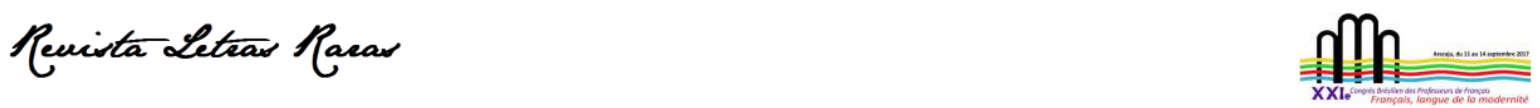

Actes du XXlème Congrès Brésilien des Professeurs De Français dans Édition spéciale de la Revue Letras Raras, 2021 ISSN : 2317-2347 - v. 10, Dossier Spécial (2021)

Todo o conteúdo da RLR está licenciado sob Creative Commons Atribuição 4.0 Internacional

séquençage des unités pédagogiques en prévoyant la répartition consciencieuse des contenus. Bien sûr, les principes régissant la perspective actionnelle, centrée sur l'établissement des tâches et visant le développement de l'apprenant en tant qu'agent, doivent prévaloir, même si on ne peut pas oublier les concepts d'éclectisme et de complexité, aptes à guider les enseignants dans le défi de choisir la meilleure approche méthodologique.

Le domaine du droit est certes très vaste et recouvre des subtilités qui sont encore plus évidentes si on compare le système juridique de deux pays différents. Par conséquent, il faut être très attentif et associer le travail de préparation des cours au travail continu de la recherche. Le progrès numérique nous a permis d'avoir accès à une quantité énorme de documents. Avec les possibilités qui en découlent, le rôle de l'enseignant s'est transformé. Sa responsabilité a augmenté. Et ses connaissances sont toujours mises à l'épreuve, en quelque sorte. L'enseignant des cours français du droit ne doit pas se borner à suivre ce qu'un manuel propose, sans s'interroger sur le contenu. II doit développer des moyens adéquats pour guider correctement un public de spécialistes. Et pour cela il est essentiel qu'il soit curieux et qu'il éprouve du plaisir à la recherche.

La différence entre les systèmes juridiques français et brésiliens révèle l'importance de l'étude de l'interculturel. L'étude de l'organisation des tribunaux, appartenant à des branches distinctes du droit, l'analyse de la procédure dans les juridictions judiciaires et dans les juridictions administratives, la compréhension du rôle joué par chaque professionnel du droit et l'examen des spécificités lexicales et discursives peuvent contribuer au perfectionnement d'apprenants désireux de développer des compétences langagières spécifiques.

\section{Références}

BERNARD, G. ; DE GUNTEN, B. ; MARTIN, A. ; NIOGRET, M. Les institutions de la France. Paris : Nathan, 2008.

CORNU, G. Vocabulaire juridique. 9ed. Paris : PUF, 2011.

MANGENOT, F. ; LOUVEAU, E. Internet et la classe de langue. Paris : Clé International, 2006.

MANGIANTE, J.-M. ; PARPETTE, C. Le français sur objectif spécifique : de l'analyse des besoins à l'élaboration d'un cours. Paris : Hachette, 2004. 

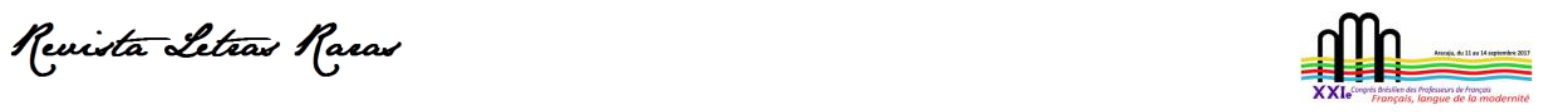

Actes du XXlème Congrès Brésilien des Professeurs De Français dans Édition spéciale de la Revue Letras Raras, 2021 ISSN : 2317-2347 - v. 10, Dossier Spécial (2021)

Todo o conteúdo da RLR está licenciado sob Creative Commons Atribuição 4.0 Internacional

MORIN, E. La complexité humaine. Paris : Flammarion, 1994.

MOURLHON-DALLIES, F. Enseigner une langue à des fins professionnelles. Paris : Didier, 2008.

PENFORNIS, J.-L. Le français du droit. Paris : Clé International, 1998.

PUREN, C. ; BERTOCCHINI, P. ; CONSTANZO, E. Se former en didactique des langues. Paris : Ellipses, 1998.

SOIGNET, Michel. Le français juridique. Paris : Hachette, 2003. 